\title{
The two-week-wait cancer initiative in urology: useful modernization?
}

\author{
Darrell Allen BSc MRCS Rick Popert MS FRCS Tim O'Brien DM FRCS
}

\section{SUMMARY}

The two-week-wait cancer initiative in the UK was designed to speed referral of patients suspected of having cancer and, by so doing, improve their outcome. We reviewed the case notes of all patients referred under this scheme to a single urology department over twelve months.

In the department, nearly one-third of new outpatient appointments came under this scheme. 124 patients were referred and all but 7 were seen within the 14-day deadline. In 62 the reason for referral was haematuria, which was macroscopic in 42. Of those with macroscopic haematuria 6 proved to have cancer, newly diagnosed in 4 ( 2 bladder, 2 renal); no patient with microscopic haematuria had cancer. Of 35 referred with a raised prostate-specific antigen 11 had prostate cancer, in most cases beyond cure. Cancer was found in 1 of 19 patients referred with a testicular mass.

In patients referred under the two-week-wait scheme with macroscopic haematuria, cancer is common.

Microscopic haematuria is seldom significant. Carcinoma of the prostate is usually advanced and beyond cure at presentation. Among patients referred with scrotal lumps, cancer of the testis is not common. The scheme as a whole is unlikely to improve cancer outcomes. Patients with macroscopic haematuria might be better served by one-stop clinics. For those with testicular lumps, most of which will be benign, the best answer might be direct general practitioner access to scrotal ultrasonography.

\section{INTRODUCTION}

Concerns over diagnostic delays in patients suspected of having cancer resulted in the Department of Health's 'two-week-wait' directive. ${ }^{1}$ It defines signs and symptoms which, if identified in primary care, warrant urgent specialist assessment. The scheme began in 1999 for patients with suspected breast cancer, ${ }^{2}$ and was extended to include urological malignant disease in 2001. Among the specified reasons for urgent urological referral are: painless macroscopic haematuria, microscopic haematuria, swelling in the body of the testis, palpable renal mass, raised age-specific prostate-specific antigen (PSA) and suspected penile cancer. No pilot study was conducted beforehand and no other country has adopted a similar scheme. ${ }^{3}$ We examined all referrals under the two-weekwait scheme to a single urology department in one year to assess its likely impact on cancer morbidity and mortality.

Department of Urology, Guy's Hospital, Thomas Guy House, St Thomas Street, London SE1 9RT, UK

Correspondence to: Mr Darrell Allen

E-mail: darrell34a@hotmail.com

\section{METHODS}

A database of all patients referred under the two-week-wait initiative to a 'spoke' district general hospital, for a 'hub' and 'spoke' urological service, was reviewed. Patients seen in the outpatients department over one year (June 2001 to May 2002) were identified and their case notes were reviewed.

\section{RESULTS}

$30 \%$ of new outpatient appointments were ring-fenced for the two-week-wait scheme and 124 patients were referred in one year, 89 male. Medical notes were unavailable for 3 . The mean age of the cohort was 66 years (range 20-96). Average time between referral and patient review was 10.3 days (range 3-15). 7 patients were not seen within the 14-day deadline.

Haematuria was the reason for referral in 62 patients, macroscopic in 42.6 of these patients were found to have cancer, of which 4 were bladder cancers and 2 renal cell carcinomas. 2 of the patients with bladder cancer had presented previously: one had a history of the disease but had been lost to follow-up whilst the other had been diagnosed abroad before seeking advice locally. Thus 4 new 
cancers were identified in the 40 patients with no previous history of urothelial cancer. All 4 bladder cancers were initially treated by transurethral resection; all proved to be superficial ( 2 pTa, 1 pTl, and 1 carcinoma-in-situ). Both patients with renal cell carcinoma underwent radical nephrectomy; in one the procedure was palliative for metastatic disease.

20 patients had microscopic haematuria and all were investigated by renal tract ultrasound and flexible cystoscopy. None proved to have cancer. In 17 no firm diagnosis was made.

The average wait for completion of basic haematuria investigations was 51 days (range 0-270). Patients with macroscopic haematuria waited an average of 41 days (0-270), whilst those with microscopic haematuria waited 70 days $(11-120)$.

35 patients were referred with a raised PSA, 27 of whom had lower urinary tract symptoms. The mean age of the cohort was 73 years (range 45-96) and median presenting PSA was $13.9 \mu \mathrm{g} / \mathrm{L}$ (range 3.4-480). 11 of these patients were diagnosed with prostate cancer. In 5 it was metastatic at presentation, mean PSA $313 \mu \mathrm{g} / \mathrm{L}$; none of these 5 underwent prostate biopsies and all were treated empirically with anti-androgens. 6 patients had T2 or T3 disease diagnosed after positive biopsies; 4 underwent external beam radiotherapy (mean PSA $32 \mu \mathrm{g} / \mathrm{L}$ [range 13.5-47.4]) and the other 2 opted for surveillance (PSAs 14.0 and $17.7 \mu \mathrm{g} / \mathrm{L})$.

19 patients were referred by their general practitioner with testicular masses, at an average age of 48 years (range 20-79). One man, aged 42, was found to have a seminoma and underwent radical orchidectomy the day after diagnosis. No other malignant disease was identified. 16 patients had scrotal ultrasound, which confirmed the clinical findings of the urologist in 15.

\section{DISCUSSION}

Though the two-week-wait scheme offers rapid access for certain patients, the priority given to this group can only be justified if substantial numbers can be shown to benefit. Ideally, the marker to examine would be survival, but this is not a realistic endpoint in a modest study from a single centre. Surrogate endpoints might include referral composition, wait to diagnosis and number of patients identified with curable disease.

To have an impact on mortality, early diagnosis must offer a better chance of cure. Of the 11 patients diagnosed with adenocarcinoma of the prostate only 4 were treated with curative intent. Even in this group cure was unlikely, because of the very high PSA at diagnosis, mean $32 \mu \mathrm{g} / \mathrm{L}$. After external beam radiotherapy
PSA was above $30 \mu \mathrm{g} / \mathrm{L} .{ }^{5}$ The two-week-wait thus seems to offer patients rapid diagnosis of advanced barely curable prostate cancer. Incidentally, none of the patients in this series were judged candidates for radical surgery.

Testicular abnormalities assessed under the two-weekwait scheme were benign in 18 of 19 cases. An alternative to the present scheme may be to offer general practitioners rapid access to scrotal ultrasonography, though this will depend on the local provision of both urological and radiological services. Definitive imaging offers reassurance to the majority and a firm diagnosis of cancer to the occasional patient, without the need for an initial urological consultation.

Microscopic haematuria accounted for $16 \%$ of all two-week-wait referrals. No cancer was detected in any patient within this cohort. The clinical significance of this feature remains controversial and some authorities suggest that it requires no investigation at all. ${ }^{6}$ If investigation is needed, urgent referral seems unnecessary.

Access to a specialist is only one step in the diagnostic pathway. Each step has the potential to delay definitive diagnosis and treatment. Speeding up one part of the process, as the two-week-wait initiative does, may not prove effective if it merely creates a new bottleneck further along the diagnostic or treatment pathway. This drawback is not theoretical. ${ }^{7}$ In our study it took over 40 days to complete basic investigations in high-risk cases of macroscopic haematuria. These patients would be far better served by a one-stop haematuria clinic where an immediate diagnosis could be established.

A concern about any 'fast track' scheme is that it has the potential to change referral patterns between primary and secondary care. When the two-week-wait initiative was instituted, projections were made for the expected ratio of benign to malignant final diagnosis in patients referred under the scheme. These projections were 6 benign referrals per cancer detected amongst haematuria referrals, 2 referrals per cancer detected in patients with a raised PSA and 10 referrals per cancer in those with suspected testicular disease. ${ }^{8}$ Our referrals yielded ratios of 10:1, 3:1 and 20:1, respectively. Thus almost twice as much benign disease is being seen as was originally intended or planned for. Clearly this has implications for the effective use of resources.

Our study did not elucidate the number of patients with newly diagnosed cancer during the year who had not been referred under the two-week-wait scheme. In planning for the future this would be an important figure to determine. Many patients with atypical signs and symptoms might be experiencing diagnostic delay because of the effect of the scheme on routine referrals. This problem has been seen in 
patients with breast cancer: half had an accelerated diagnosis and the other half a delayed diagnosis. ${ }^{9}$

There is a further area of concern about the initiative, which could reduce efficiency and possibly even delay cancer diagnosis in many patients: clinicians have previously used the gap between referral and consultation to organize relevant investigations. Pre-clinic investigations allow for a one-stop approach to disease management and reduce the need for follow-up. The very short period allowed between referral and consultation as part of the initiative has meant that none of the patients are pre-investigated. This is particularly relevant in patients with scrotal disease and haematuria, where imaging or urinary cytology tests are frequently a prerequisite for diagnosis.

In conclusion, this study demonstrates that the twoweek-wait criteria, even when fully met, are unlikely to improve survival in urological cancer and may not be the most efficient and cost-efficient route to diagnosis.

\section{REFERENCES}

1 Department of Health. The New NHS, Modern and Dependable. London: Stationery Office, 1997

2 NHS Executive. Breast Cancer Waiting Times-Achieving the Two-week Target. London: Department of Health, 1998

3 Cant PJ, Yu DSL. Impact of the '2 week wait' directive for suspected cancer on service provision in a symptomatic breast clinic. Br J Surg 2000;87:1082-6

4 Pisansky TM, Cha SS, Earle JD, et al. Prostate specific antigen as a pretherapy prognostic factor in patients treated with radiation therapy for clinically localised prostate cancer. J Clin Oncol 1993;11:2158-63

5 Zagars GK. Prostate specific antigen as an outcome variable for T1 and T2 prostate cancer treated by radiation therapy. J Urol 1994;152: 1786-91

6 Malstrom P. Time to abandon testing for microscopic haematuria in adults? BMJ 2003;326:813-15

7 Walsh S, Bruce C, Bennington S, Ravi S. The fourteen day rule and colorectal cancer. Ann R Coll Surg Engl 2002;84:386-8

8 Department of Health. Referral Guidelines for Suspected Cancer. London: Stationery Office, 2000

9 Khawaja AR, Allan SM. Has the breast cancer 'two week wait' guarantee for assessment made any difference? Europ J Surg Oncol 2000;26:536-9 